\title{
Coloration and Functional Modification of Silk Fiber via Dopamine Polymerization
}

\author{
Xiao-Hui Kuang ${ }^{\mathrm{a}}$, Jin-Ping Guan ${ }^{\mathrm{b}}$, Wei Chen ${ }^{\mathrm{c}}$, Ren-Cheng Tang $^{\mathrm{d}}$ and \\ Guo-Qiang Cheng ${ }^{\mathrm{e}}$ \\ College of Textile and Clothing Engineering, Soochow University, Suzhou 215123, China \\ a1041250216@qq.com, bguanjinping@suda.edu.cn, 'chen0376@126.com, \\ dtangrencheng@suda.edu.cn, êchenguoqiang@suda.edu.cn
}

\begin{abstract}
The oxidative polymerization of dopamine for the functional surface modification of materials has drawn extensive attention. In this work, the coloration and functional modification of silk fiber by dopamine polymerization were studied. The conditions of dopamine application were first discussed in the absence of specific oxidants, and then the surface morphology, color characteristics, color fastness, stability to acid and alkali, UV protection ability and antistatic property of the colored and modified silk fiber were determined. The $\mathrm{pH}$, temperature and time of dopamine application were found to exert great influence on the color depth of silk fiber. A weakly alkaline condition ( $\mathrm{pH}$ 8.5) was an essential prerequisite. The treated silk fiber showed a brown grey color, a high color fastness to washing, and a good stability to acid and weak alkali. The polymerization of dopamine on the surface of silk fiber imparted UV protection and antistatic effects to silk fabric.
\end{abstract}

Keywords: silk, coloration, functional modification, dopamine, polymerization.

\section{Introduction}

Dopamine (3,4-dihydroxyphenylethylamine), a small molecule compound, contains a catechol structure with one amine group attached via an ethyl chain, and it can easily be oxidized and subsequently self-polymerize in the presence of weakly alkaline agents, and oxygen or oxidants [1]. The resulting polydopaime possesses excellent biocompatibility and hydrophilicity, and can assemble other functional molecules as the secondary reaction platform [2]. The oxidation polymerization of dopamine has been applied to the surface coatings of a wide variety of materials (such as polymers, metals and ceramics). In recent years, the functional surface coatings of textile fibers via dopamine polymerization has attracted a lot of attention and become a hot research topic. In this regard, most of reports focused on the use of polydopamine coating as the binder substrate for the adhesion of inorganic nanomaterials on the surface of fibers [3-4]. In addition to this, dopamine polymerization was also used to induce the surface coloration of cotton, wool and polybenzimidazole fibers through a simple dip-coating procedure in the presence of specific oxidants [1]. A recent study reported the laccase-mediated in situ oxidation of dopamine for the bioinspired coloration of silk fabric [5]. In this work, the self-polymerization of dopamine was applied to the coloration and functional modification of silk fiber by a dipping approach. The conditions of dopamine application as well as the surface morphology, color characteristics, color fastness, stability to acid and alkali, UV protection ability and antistatic property of the colored and modified silk fiber were studied.

\section{Materials and Methods}

\subsection{Materials}

The scoured silk habotai fabric (warp and weft count, 23.3 dtex/2; warp density, 42 threads/cm, and weft density 60 threads/cm; weight per unit area, $52 \mathrm{~g} / \mathrm{m}^{2}$ ) was purchased from Huasi Silk Printing and Dyeing Co. Ltd., China. Dopamine hydrochloride with a purity of $98.5 \%$ and tris(hydroxymethyl)aminomethane hydrochloride (Tris-HCl) was supplied by Yuanye 
Biotechnology Co. Ltd., China. Tris(hydroxymethyl) aminomethane (Tris) was purchased from Sangon Biotech Co. Ltd., China.

\subsection{Coloration and Functional Modification of Silk Fabric by Dopamine}

The coloration and functional modification of silk fabric was performed by dipping silk in the dopamine hydrochloride solution using a liquor ratio of 80:1. The $\mathrm{pH}$ of solution was adjusted with Tris and Tris-HCl. All the treatments were performed at a required constant temperature for a certain period of time. After treatment, the sample was washed with running water, and dried in an oven. To assess the effect of temperature, six temperatures (30, 35, 40, 45, 50 and $55^{\circ} \mathrm{C}$ ) were employed, and the other parameters were: $2 \mathrm{~g} / \mathrm{L}$ dopamine, $\mathrm{pH} 8$ and $24 \mathrm{~h}$. To estimate the effect of $\mathrm{pH}$, the $\mathrm{pH}$ of the solution was adjusted to 7.0-9.5, and $2 \mathrm{~g} / \mathrm{L}$ dopamine, $45^{\circ} \mathrm{C}$ and $24 \mathrm{~h}$ were used. To study the effect of dopamine dosage, five dopamine dosages (1, 2, 3, 4 and $5 \mathrm{~g} / \mathrm{L}$ ) were used, and the other parameters were as follows: $\mathrm{pH} 8.5,45^{\circ} \mathrm{C}$ and $24 \mathrm{~h}$. To evaluate the effect of time, $2 \mathrm{~g} / \mathrm{L}$ dopamine, $\mathrm{pH} 8.5$ and $45{ }^{\circ} \mathrm{C}$ were used, and silk fabric was treated for 2 to $30 \mathrm{~h}$. These discussions gave the optimized conditions of dopamine application: $\mathrm{pH} 8.5,45^{\circ} \mathrm{C}$ and $24 \mathrm{~h}$. Thus, the silk fabric treated with $2 \mathrm{~g} / \mathrm{L}$ dopamine was used for the evaluations of color characteristics, color fastness, stability to acid and alkali, and functionalities.

\subsection{Measurements}

The color parameters of silk fabric including lightness $\left(\mathrm{L}^{*}\right)$, chroma $\left(\mathrm{C}^{*}\right)$, hue $(\mathrm{h})$, redness-greenness value $\left(a^{*}\right)$, yellowness-blueness value $\left(b^{*}\right)$, and color strength $(K / S)$ were measured using a HunterLab UltraScan PRO reflectance spectrophotometer (illuminant D65; $10^{\circ}$ standard observer). The color fastness to washing with soap of silk fabric was tested as per GB/T 3921-2008. The stability to acid and alkali of silk fabric was evaluated using the following method: the fabric was immersed into the acidic and alkaline solutions using a liquor ratio of 100:1, whose $\mathrm{pH}$ was adjusted to 2, 5, 9 and 12 with hydrochloric acid and sodium hydroxide, and treated at $50{ }^{\circ} \mathrm{C}$ for $60 \mathrm{~min}$; afterwards, the sample was removed, washed in distilled water, and allowed to dry in an oven. The surface morphology of silk fiber was observed using a Hitachi TM3030 tabletop scanning electron microscope (SEM). The ultraviolet protection factor (UPF) and UV transmittance of silk fabric were determined by a Labsphere UV-1000F ultraviolet transmittance analyzer. The antistatic property of silk fabric was evaluated as per GB/T 12703.1-2008.

\section{Results and Discussion}

\subsection{Conditions of Application of Dopamine to the Coloration and Modification of Silk Fabric}

Four factors affecting the dopamine modification of silk fabric were temperature, $\mathrm{pH}$, dopamine dosage and time. The color depth of the treated sample was used as the evaluation criterion. Fig. 1a shows the effect of temperature. The color depth of silk fabric increased gradually with increasing temperature up to $45{ }^{\circ} \mathrm{C}$, and the extremely limited increment of color depth occurred with further increasing temperature. The increased color depth is induced by the increased motion energy of dopamine and the enhanced self-polymerization ability of dopamine that was accompanied by the evaluated temperature. Fig. 1b shows that silk fabric exhibited the highest color depth when treated at $\mathrm{pH}$ 8.5, which is in agreement with most reported synthesis of polydopamine [6]. In acidic media, dopamine is stable toward oxidation in the absence of metal ions and specific oxidants, and a weakly alkaline condition is a fundamental requirement for the self-polymerization of dopamine [6]. Fig. 1c shows that $2 \mathrm{~g} / \mathrm{L}$ dopamine enabled the color of silk fabric to become the darkest. Fig. 1d shows that the color depth of silk fabric increased gradually with an increase in time, and reached to the highest value at 24 h. Fig. 2 displays the deposition of polydopamine on the silk surface. As shown in Fig. 2, the deposition amount increased gradually with increasing time, which supports the results of Fig. 1d. This observation indicates that the self-polymerization of dopamine is very slow in the absence of specific oxidants.

\subsection{Color Characteristics of the Colored and Modified Silk Fabric}

Fig. 3 shows the color characteristics and visual photos of the silk fabric treated without and with dopamine. The untreated silk had very low $a^{*}, b^{*}$ and $C^{*}$ values with a very high $L^{*}$ because of its 
white color. The treated silk displayed low $\mathrm{a}^{*}, \mathrm{~b}^{*}$ and $\mathrm{C}^{*}$ values with a low $\mathrm{L}^{*}$ value, indicating that its color is dull and dark. The treated silk had a hue angle of $68.91^{\circ}$, and its visual color was brown grey. Moreover, in the UV-visible absorption spectrum of the treated silk fabric, a sharp absorption band was not found in the visible light range. This is a great difference between polydopamine and synthetic dyes.
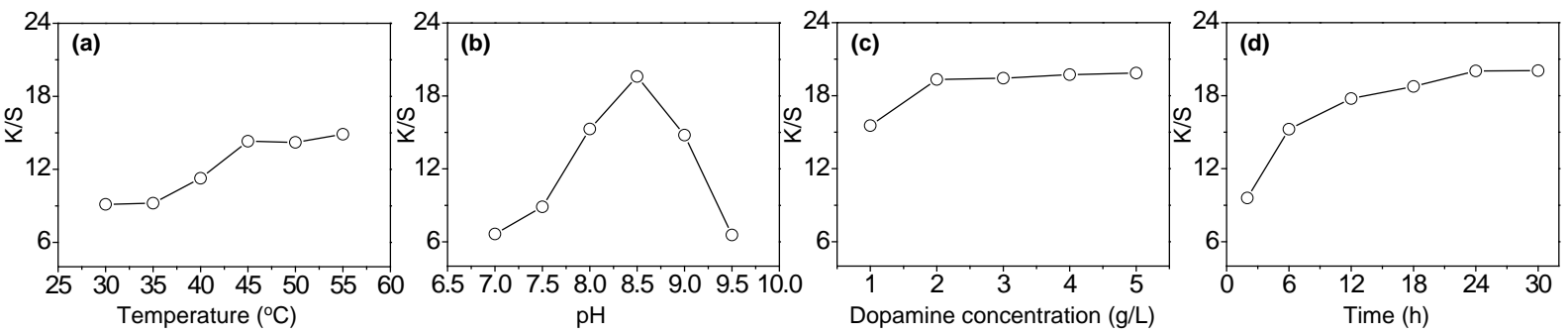

Fig. 1 Color depth of the silk fabrics treated with dopamine in different conditions: (a) temperature, (b) $\mathrm{pH}$, (c) dopamine, and (d) time

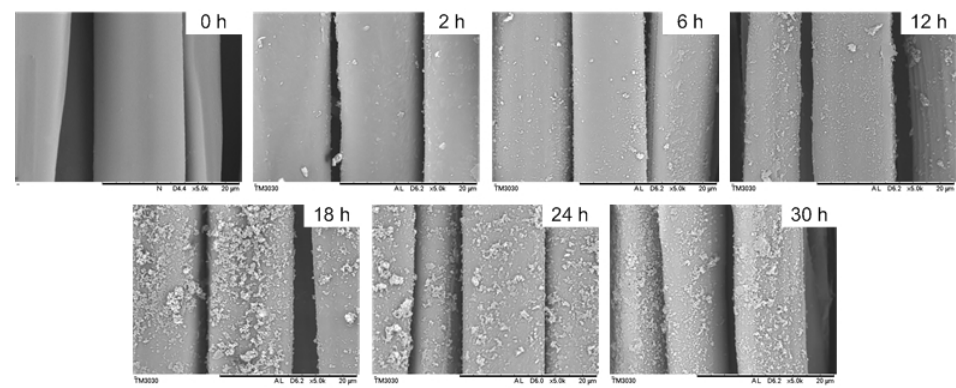

Fig. 2 SEM images of the silk fabrics treated with dopamine for different times

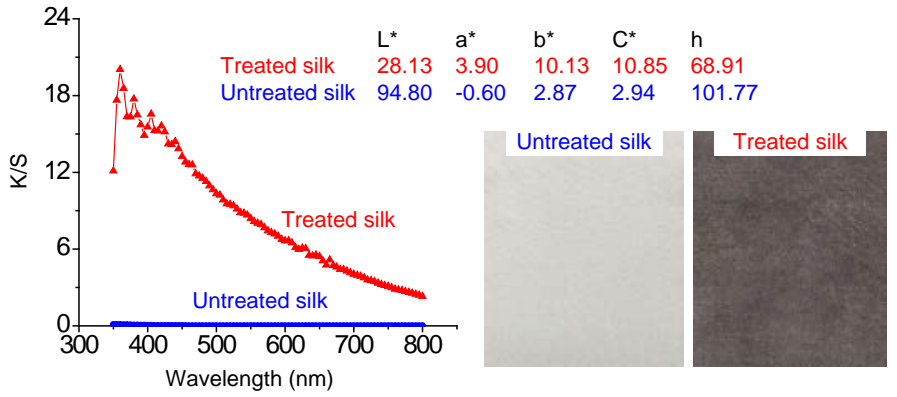

Fig. 3 Color characteristics and visual photos of the silk fabrics treated without and with dopamine

\subsection{Color Fastness and Stability of the Colored and Modified Silk Fabric}

Fig. 4 shows the color fastness to washing with soaping, and stability to acid and alkali of the silk fabric treated with dopamine. Interestingly, the treated silk exhibited the highest fastness level (Fig. $4 a)$, regardless of color change and staining. Even after the treated silk was subjected to repeated washing, it still possessed excellent washing fastness. This suggests that polydopamine has a strong adhesion force towards the surface of silk fiber. Fig. 4b shows the change in the color depth of the treated silk fabric subjected to the treatment in hydrochloric acid and sodium hydroxide solutions, whose $\mathrm{pH}$ values were adjusted by acid and alkali dosages. Clearly, the treatment at the $\mathrm{pH}$ ranging from 2 to 9 had no impact on the color depth of silk fabric. However, the treatment at pH 12 increased the color depth. This phenomenon should be due to the ionization of phenolic hydroxyl groups in polydopamine in a relatively strong alkali media.

\subsection{Functional Properties of the Colored and Modified Silk Fabric}

Table 1 shows the functional properties of the silk fabric treated with dopamine. The untreated sample had poor UV protection ability as indicated by high UV transmittance and low UPF. Compared with the untreated sample, the treated silk fabric displayed markedly reduced transmittance of UVA and UVB radiation, and as a result its UPF increased obviously. Thus the 
treated silk fabric exhibited UV protection effect. It is well known that silk fiber has good antistatic performance because of its hygroscopic nature. After treated with dopamine, the silk fabric showed better antistatic ability that was verified by a very short static half period.
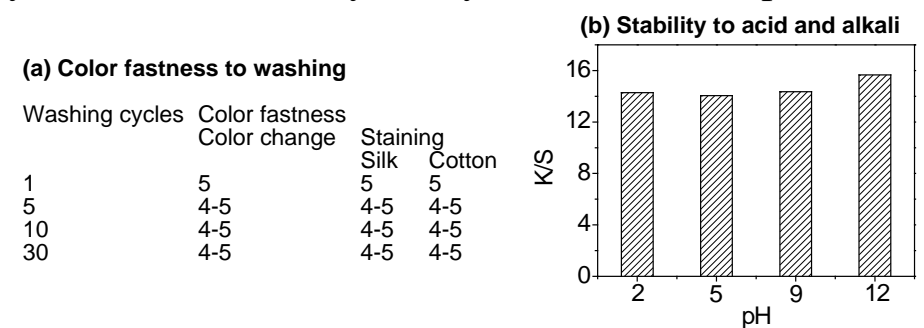

Fig. 4 Color fastness (a) and stability to acid and alkali (b) of the silk fabric treated with dopamine

Table 1 UV protection and antistatic properties of the silk fabrics treated without and with dopamine

\begin{tabular}{|c|c|c|c|c|c|}
\hline \multirow{2}{*}{ Sample } & \multicolumn{3}{|c|}{ UV protection } & \multicolumn{2}{c|}{ Antistatic ability } \\
\cline { 2 - 6 } & UPF & $\mathrm{T}_{\text {UVA }}(\%)$ & $\mathrm{T}_{\text {UVB }}(\%)$ & Electrostatic voltage (V) & Static half period (s) \\
\hline Untreated & 2.77 & 51.83 & 27.91 & 1180 & 35.57 \\
\hline Treated & 13.84 & 7.83 & 7.12 & 1160 & 1.52 \\
\hline
\end{tabular}

\section{Conclusions}

In this work, the self-polymerization of dopamine was applied to the coloration and functional modification of silk fabric. The $\mathrm{pH}$ value of dopamine solution was found to have the greatest impact on the color depth of silk fabric, and a weakly alkaline condition was a fundamental requirement. In addition, temperature and time were also two important application conditions of dopamine. The self-polymerization of dopamine on the surface of silk fiber was very slow. The self-polymerization of dopamine enabled silk fabric to become a brown grey color with high color fastness to washing and good stability to acid and weak alkali, and also provided UV protection and antistatic effects.

\section{Acknowledgments}

This study was funded by the Priority Academic Program Development (PAPD) of Jiangsu Higher Education Institutions (No. 2014-37).

\section{References}

[1] L. He, V.L.L. So, J.H. Xin, Dopamine polymerization-induced surface coloration of various materials, RSC Adv. 4 (2014) 20317-20322.

[2] H. Lee, S.M. Dellatore, W.M. Miller, P.B. Messersmith, Mussel-inspired surface chemistry for multifunctional coatings, Science. 318 (2007) 426-430.

[3] Z. Lu, J. Xiao, Y. Wang, M. Meng, In situ synthesis of silver nanoparticles uniformly distributed on polydopamine-coated silk fibers for antibacterial application, J. Colloid Interf. Sci. 452 (2015) 8-14.

[4] J. Yang, H. Xu, L. Zhang, Y. Zhong, X. Sui, Z. Mao, Lasting superhydrophobicity and antibacterial activity of $\mathrm{Cu}$ nanoparticles immobilized on the surface of dopamine modified cotton fabrics, Surf. Coat. Tech. 309 (2017) 149-154.

[5] W. Jia, Q. Wang, X. Fan, A. Dong, Y. Yu, P. Wang, Laccase-mediated in situ oxidation of dopa for bioinspired coloration of silk fabric, RSC Adv. 7 (2017) 12977-12983.

[6] W. Zheng, H. Fan, L. Wang, Z. Jin, Oxidative self-polymerization of dopamine in an acidic environment, Langmuir. 31 (2015) 11671-11677. 